\title{
Una bella relación entre la conjetura de Golbach y el teorema de Dirichlet
}

\author{
A beautiful relationship between Goldbach guess and theorem Dirichlet \\ CAMPO ELÍAS GONZALEZ PINEDA. \\ Departamento de Matemáticas, Universidad Tecnología de Pereira, Pereira, Colombia. \\ Correo: cegpeutp.edu.co
}

\begin{abstract}
Resumen- En este artículo se muestra el proceso que se llevó a cabo para encontrar una importante relación entre la Conjetura de Goldbach y el teorema de Dirichlet. Quizá podría suprimirse la primer parte de este artículo, pero no se hizo con el fin de mostrar el proceso histórico que se recorrió para llegar al resultado. Se espera que los resultados presentados muestren en realidad el esfuerzo hecho para alcanzar una prueba que duró muchos años en lograrse.
\end{abstract}

Palabras clave - Número primo, conjetura, Goldbach, Dirichlet.

Abstract - This article describes the process that was undertaken to find an important relationship between the Goldbach Conjecture and Dirichlet theorem. Perhaps it could be suppressed the first part of this article, but I do not know made in order to show the historical process that he ran to get the result. It is hoped that the results presented actually show the effort made to attain an test that lasted many years in achieved.

Key Word — Prime Number, conjecture, Goldbach, Dirichlet

\section{INTRODUCCIÓN}

Este artículo muestra como a veces las cosas complejas no dejan ver las simples. El resultado fundamental acerca de la conjetura de Goldbach, se deduce de unos resultados muy simples y del Teorema de Dirichlet para números primos: Si $a, b$ son enteros primos relativos la sucesión $p=a n+b$ contiene infinitos números primos. La justificación de este resultado es muy difícil y requiere de la variable compleja. Aquí se generaliza este resultado y además se utiliza para probar de manera sencilla el postulado de Bertrand, que afirma que entre $n$ y $2 n$ hay un primo $p$. Al final en realidad se resume que todo está relacionado, solo es buscar los caminos adecuados para ver las relaciones entre situaciones aparentemente inconexas. Espero que lo realizado aquí sirva como motivación para matemáticos inquietos que busquen herramientas simples para probar resultados aparentemente difíciles de mostrar
- No se hacen referencias históricas de la conjetura porque se considera que hay ya suficientes en la literatura.

\section{CONTENIDO}

A. La conjetura de Goldbach

La Conjetura de Goldbach afirma que todo número par mayor o igual a 4 se puede expresar como la suma de dos números primos. Se ha comprobado por cálculo directo, que esto es cierto para un número suficiente de casos que hacen pensar que la conjetura es cierta. En este documento mostramos una conjetura equivalente y veremos como la conjetura y el Teorema de Dirichlet está relacionados.

De otra parte, es claro que si $q$ es un número primo y $P=2 q$, entonces $P$ es un número par. Así, al haber infinitos números primos existen infinitos pares que se pueden escribir como la suma de dos números primos. Más aún, sabemos que todo primo distinto de 2 es impar, y como suma de impares da par, el número de pares que se pueden escribir como suma de dos primos aumenta. Por ahora analicemos algunos casos en los que se comprueba la conjetura por cálculo directo.

$$
\begin{array}{ll}
4=2+2 & 16=13+3=11+5 \\
6=3+3 & 24=17+7=19+5 \\
8=5+3 & 80=73+7=37+43 \\
10=5+5=7+3 & 8900=7+8893=13+8887 . \\
12=7+5 & 14=7+7=11+3
\end{array}
$$

Una sencilla observación a la lista anterior muestra que los números primos involucrados para algunas representaciones no son necesariamente únicos, como en el caso del número 14. Es decir, la representación en suma de primos no es única en 
general. Antes de continuar, introduzcamos la siguiente definición.

DEFINICIÓN 1.1 Un entero positivo par se llama de Goldbach si se puede escribir como la suma de dos números primos.

Veamos qué conclusiones podemos obtener suponiendo que la conjetura fuera cierta. Es decir, supongamos que todo par mayor o igual a 4 se puede escribir como la suma de dos números primos. En particular el caso $P=4$ se cumple trivialmente.

Observemos que los números pares son de la forma $A=2 k$, donde $k$ puede ser par o impar. Analicemos las siguientes situaciones:

Sea $P>4$ un número par dado y supongamos que

$2 P=p_{1}+p_{2}$, con $p_{1}, p_{2}$ números primos. Debe existir un impar I de tal manera que:

$$
2 P=P+I+P-I=p_{1}+p_{2}
$$

donde

$$
P+I=p_{1}, \text { y } P-I=p_{2} .
$$

De igual forma, sea $I>1$ un impar dado y supongamos que $2 I=p_{1}+p_{2}$, con $p_{1}, p_{2}$ números primos. Debe existir un par $\mathrm{P}$ de tal manera que:

$$
2 I=I+P+I-P=p_{1}+p_{2}
$$

donde

$$
P+I=p_{1}, \text { y } I-P=p_{2}
$$

Para el caso (1.1) es fácil ver que los primos son de la forma $p_{1}=4 m+1$ y $p_{2}=4 n-1$. Así $P=2(m+n)$ y los primos para el caso (1.2) son de la forma $p_{1}=4 m \pm 1, p_{2}=4 n \pm 1 \mathrm{e}$ $I=2(m+n) \pm 1$

Podemos de esta observación, obtener varios resultados que consideramos continuación.

\section{TEOREMA 1.1}

1. Sean $m, n$ enteros positivos de tal manera que

$$
p_{1}=4 m+1, p_{2}=4 n-1 \text {, }
$$

sean números primos. Hagamos $P=2(m+n)$, entonces se tiene que

$$
2 P=p_{1}+p_{2}
$$

2. Sean $m, n$ enteros positivos de tal manera que

$$
p_{1}=4 m+1, \quad p_{2}=4 n+1 \quad\left(o p_{1}=4 m-1,\right.
$$
$\left.p_{2}=4 n-1\right)$ sean números primos. Hagamos $I=2(m+n)+1(I=2(m+n)-1)$, entonces se tiene que:

$$
2 I=p_{1}+p_{2}
$$

DEMOSTRACIÓN. Para la parte (1), hacemos $I=2(m-n)+1$, luego $2 P=P+P=P+I+P-I=p_{1}+p_{2}$

Como existen infinitos números primos de la forma $4 l \pm 1$ tenemos el siguiente resultado como Corolario del anterior.

\section{TEOREMA 1.2}

1. Existen infinitos números de Goldbach de la forma $2 P$, con $P$ par.

2. Existen infinitos números Goldbach de la forma $2 I$, con $I$ impar.

Se trata entonces de ver si todo entero par positivo es de Goldbach. La discusión anterior nos permite enunciar la siguiente conjetura que es equivalente a la de Goldbach.

CONJETURA 1. Sea $P$ un par (impar). Entonces, existe un impar (par) $I<P$ primo relativo con $P$ de tal manera que

$$
P+I=p_{1} \text { y } P-I=p_{2}
$$

donde $p_{1}, p_{2}$ son números primos.

Para el caso impar, si éste es primo se puede elegir el par como cero. Nótese que efectivamente si se cumple (1.3) también se cumple la Conjetura de Goldbach y viceversa, basta sumar las dos igualdades.

El recíproco de la Conjetura 1 se puede probar teniendo en cuenta la siguiente propiedad interesante de los números impares.

\section{TEOREMA 1.3}

1. Sean $a, c$ enteros impares positivos, entonces:
a) $\frac{a+c}{2}$ es par si y sólo si $\frac{a-c}{2}$ es impar.
b) $\frac{a+c}{2}$ es impar si y sólo si $\frac{a-c}{2}$ es par.

2. Si $a, c$ son pares $\frac{a+c}{2} y \frac{a-c}{2}$ tienen la misma paridad. 
La demostración del resultado anterior es elemental y se deja al lector ([2]). De este resultado tenemos el siguiente corolario.

\section{COROLARIO 1.1}

Sean $p_{1}, p_{2}$ impares positivos. Si $P=\frac{p_{1}+p_{2}}{2}$ es par (impar) existe un impar (par) I de tal manera que

$$
P+I=p_{1} \text { y } P-I=p_{2}
$$

En particular, si $p_{1} p_{2}$ son números primos y en este caso es fácil verificar que $P$ e $I$ tienen que ser primos relativos.

La conjetura 1 puede escribirse también de la siguiente manera:

CONJETURA 2. Para todo entero $l \geq 2$ existen $m, n, a, b$ enteros positivos tales que $l=m+n=a+b$ donde

1. $p_{1}=4 m+1, p_{2}=4 n-1$ son números primos.

2. $p_{1}=4 a+1, p_{2}=4 b+1$ son números primos o

$p_{1}=4 a-1, p_{2}=4 b-1$ son números primos.

Para ver esto se supone cierta la conjetura 1 se suma y resta para el caso par e impar y se obtiene el resultado.

Veamos ahora como está involucrado el teorema de Dirichlet en todo este asunto.

El teorema de Dirichlet, postulado de Bertrand, Goldbach.

OBSERVACIÓN: Recordemos que todo impar es de la forma $4 k+1$ o de la foma $4 k-1$ pero no de ambas a la vez. Como todo primo distinto de 2 es impar, entonces todo primo impar es de esta forma. Más aún se puede demostrar con relativa facilidad que existen infinitos primos de la forma $4 k+1$ e infinitos de la forma $4 l-1$. Es más, hay tantos primos impares de la forma $4 k+1$ como de la forma $4 l-1$. Como veremos, en realidad este es un caso particular de la generalización del Teorema de Dirichlet que estudiamos a continuación.

Nota: $((X, Y))$ indica máximo común divisor.

Recordemos que el Teorema de Dirichlet afirma que si $a, b$ son primos relativos, entonces la sucesión

$$
p(n)=a n+b
$$

contiene infinitos números primos. Considerando todas las posibilidades para $a$ y $b$ en la ecuación (1.4) vemos que el Teorema de Dirichlet se reduce al siguiente resultado.

TEOREMA 1.4 La sucesión $p(n)=2 t n+I,((2 t, I))=1, I \in\{1,3,5, \ldots, 2 t-1\}$

contiene infinitos números primos. (2t es un par positivo cualquiera pero fijo.)

Para la demostración de este resultado ver la referencia [2].

Observaciones sencillas de (1.4) y (1.5) cuando son números primos muestran que $((a n, b))=1,((n, b))=1,((2 t n, I))=$ $1,((n, I))=1$. El Teorema (1.4) se puede generalizar fácilmente así.

\section{TEOREMA 1.5 Elías}

Para cada $n \geq 2$ existen $I, I^{\prime} \in\{1,3,5, \ldots, 2$ tn -1$\}$ de tal manera que:

1. $p(n)=2 t n+I$ es un número primo para algún $I$.

2. $p(n)=2 t n-I^{\prime}$ es un número primo para algún $I^{\prime}$.

DEMOSTRACIÓN. Hacemos la primera parte por inducción y es claro que la parte dos es igual. Para simplificar hagamos

$t=2$. Es decir consideremos la sucesión

$$
p(n)=4 n+I
$$

Si $n=2, p(2)=8+I, I \in\{1,3,5,7\}$, vemos que $I=3$ o $I=5$ hacen cierta la proposición. Supongamos que el resultado es cierto para $n$. Es decir $p(n)=4 n+I$ es un número primo para algún impar $I<4 n$.

Ahora,

$$
p(n+1)=4(n+1)+I_{1}=4 n+4+I_{1}, 4+I_{1}<4(n+1) .
$$

Por hipótesis de inducción $4 n+I$ es primo para algún

$I<4 n$. Podemos hacer

$$
4+I_{1}=I<4 n<4(n+1)
$$

y el resultado es cierto para todo $n$.

De otro lado si hacemos

$$
p(n)=2 t n+I
$$

Vemos que $p(2)=4 t+I$ es primo para algún $I<4 t$ por lo que acabamos de demostrar. Si suponemos que

$$
p(n)=2 t n+I
$$

es primo para algún $I<2 t n$ y hacemos 


$$
p(n+1)=2 t(n+1)+I_{1}=2 t n+2 t+I_{1}
$$

es primo aplicando la Hipótesis de inducción con $2 t+I_{1}=I$.

En particular si $t=1$ entonces

1. $p(n)=2 n+I$ es un número primo para algún impar $I<2 n$.

2. $\quad p(n)=2 n-I^{\prime}$ es un número primo para algún impar $I^{\prime}<2 n$.

Nota: El I que aparece en 1. y 2. del teorema 1.5 No tiene que ser el mismo, nuestro objetivo es mostrar que existe el mismo en las dos situaciones.

Obsérvese que para la parte (1) del teorema $((2 t n, I))=1$, para la parte dos esto no es cierto en general como es el caso $2(9)-15=3$. Para ilustrar un poco los resultados anteriores consideremos el siguiente caso particular.

EJEMPLO 1.1. Sea $p(n)=4 n+I$, entonces

1. $p(2)=8+I, I \in\{1,3,5,7\}$.

2. $p(3)=12+I, I \in\{1,5,7,11\}$

3. $p(4)=16+I, I \in\{1,3,5,7,9,11,13,15\}$.

4. $p(5)=20+I, I \in\{1,3,7,9,11,13,17,19\}$. En particular $p(5)=20+I=16+4+I$ y hacemos $4+I=7$ о $I=3$.

5. La expresión $200-I$ con $I<200$ impar contiene todos los primos menores o iguales a 200, y la expresión $200+I$ contiene todos los primos menores que 400 y mayores que 200 .

De otra parte, si $I$ es un impar y $P<I$ es un par, podemos considerar la sucesión

$$
p(n)=\operatorname{In}+P
$$

Si $P(n)$ es primo vemos que $n$ tiene que ser impar, digamos $n=2 m+1$. Es decir, (1.6) queda en la forma

$$
p(m)=I(2 m+1)+P=2 t^{\prime} m+I^{\prime}, t^{\prime}=I, I^{\prime}=I+P
$$

Obsérvese que $I^{\prime}<2 t^{\prime}$ ya que $P<I$ entonces $P+I<2 I$. De otro lado si hacemos

$$
p(n)=I n-P
$$

Como $-P<I$ vemos que $I-P<2 I$, luego si $n=2 m+1$ tenemos que (1.7) se convierte en

$$
p(m)=I(2 m+1)-P=2 t^{\prime} m+I^{\prime}, t^{\prime}=I, I^{\prime}=I-P
$$

Se tiene entonces el siguiente corolario.

COROLARIO 1.2 Sea $I$ un impar dado. Para todo $n \geq 5$ impar, existe un par $P, P^{\prime}<\operatorname{In} \operatorname{con}((\operatorname{In}, P))=1,\left(\left(\operatorname{In}, P^{\prime}\right)\right)=$ 1 de tal manera que

$$
p_{1}=\operatorname{In}+P, p_{2}=\operatorname{In}-P^{\prime}
$$

son números primos.

EJEMPLO 1.2 Si hacemos $I=1$ se tiene que $n \geq 5$ impar, es decir, tenemos

$$
n+P, n-P^{\prime}, P, P^{\prime} \in\{0,2, \ldots, n-1\}
$$

Hagamos $n=2 m+1$ entonces la expresión se convierte en

$$
\begin{gathered}
p(m)=2 m+1+P, q(m)=2 m-\left(P^{\prime}-1\right), \\
P, P^{\prime} \in\{0,2, \ldots, n-1\} \\
p(m)=2 m+I, q(m)=2 m-I^{\prime}, \\
I, I^{\prime} \in\{1,3, \ldots, 2 m-1\}
\end{gathered}
$$

De lo visto hasta ahora, vemos que el Teorema de Dirichlet implica la conjetura de Goldbach. Más aún, si consideramos las sucesiones

$$
p(n)=2 n+I, \quad I<2 n, \quad q(n)=2 n-I^{\prime}, \quad I^{\prime}<2 n
$$

donde $p(n), q(n)$ son números primos, notamos que

$$
\begin{gathered}
I \in H=\{1,3,5, \ldots 2 n-1\} \\
I^{\prime} \in W=\{1,3,5, \ldots 2 n-1\}
\end{gathered}
$$

Para algún $n$ debe ser que $I=I^{\prime}$. De igual forma en la sucesiones

$$
p(n)=n+P, P<n, \quad q(n)=n-P^{\prime}, P^{\prime}<n
$$

donde $p(n), q(n)$ son números primos, notamos que

$$
\begin{gathered}
P \in H=\{2,4,6, \ldots n-1\} \\
P^{\prime} \in W=\{2,4,6, \ldots n-1\}
\end{gathered}
$$

Para algún $n$ debe ser que $P=P^{\prime}$. Esto se debe a que en la expresión $P-I$ es imposible que se encuentren primos 
únicamente cuando $P$ e $I$ no sean primos relativos. Para tal efecto veamos el siguiente resultado.

TEOREMA 1.6 Supongamos que $((2 n, I))=l \neq 1$ y que $p_{1}=2 n-I$ es un número primo. Entonces $p_{1}=l$.

DEMOSTRACIÓN. Con las hipótesis dadas tenemos que $2 n=l k, I=k^{\prime} l$, por lo que

$$
2 n-I=l k-l k^{\prime}=l\left(k-k^{\prime}\right)=p_{1}
$$

Como $p_{1}$ es primo se deduce que $k-k^{\prime}=1 \mathrm{y}$ por tanto $p_{1}=$ $l, k=k^{\prime}+1$.

EJEMPLO 1.3 $\mathrm{Si}((2 n, I))=p_{2} \quad \operatorname{con} p_{2}=2 n-I \quad$ primo entonces $p_{1}=2 n+I$ no es primo, ya que $p_{2} / p_{1}$.

TEOREMA 1.7 Para cada $n \geq 2$ existe un impar $I<2 n$ de tal manera que $((2 n, I))=1$ y además $p_{1}=2 n-I$ es un número primo.

DEMOSTRACION. La demostración es por inducción. En efecto, haciendo $p(n)=2 n-I, I<2 n$. Si $n=2$, tenemos $p(n)=4-I, I \in\{1,3\},$,$\quad vemos que I=1$, hace cierta la proposición. Supongamos que el resultado se cumple para $n$ y veamos que se cumple para $n+1$. En efecto,

$$
p(n+1)=2(n+1)-I=2 n-I_{1}, \quad I_{1}=I-2 .
$$

Por H.I existe $I_{1}<2 n$ impar talque

$$
p(n+1)=2(n+1)-I=2 n-I_{1}
$$

es un número primo, como $I_{1}=I-2$ vemos que $I=I_{1}+2 \mathrm{y}$ es resultado es cierto para todo $n \geq 2$. Nótese que: $I<2(n+1)$. De otro lado note que si $d=((2 n+2, I))$ se tiene que $d /(2 n+2-I)=2 n-I_{1}$, pero $2 n-I_{1}$ es primo, por tanto $d=1$ o $d=2 n-I_{1}$, pero lo último no puede ser y así $d=1$. Es decir, $2(n+1)$ e $I$ son primos relativos.

Del teorema 1.5 y el corolario 1.2 se deduce el famoso postulado de Bertrand que afirma que existe un primo $p$ entre $n$ y $2 n$. Es decir:

\section{Postulado de Bertrand}

Para todo $n \geq 3$ existe almenos un primo $p$ tal que

$$
n<p<2 n \text {. }
$$

DEMOSTRACION Por el teorema 1.5 con $t=1$ Supongamos que $p=2 n+I, I<2 n$ es un número primo. Sumando $2 n$ es claro que $2 n+I<4 n$ luego

$$
2 n<2 n+I<4 n \text {. }
$$

De otra parte, por el corolario 1.2 tenemos que si

$$
p=\operatorname{In}+P, \quad P<\operatorname{In}
$$

es primo ( $n$ es impar) entonces se tiene que $P<$ In implica

$$
\text { In }<\text { In }+P<2 \text { In }
$$

En particular si $I=1$ se encuentra

$$
n<I+P<2 n, n \geq 3 \text {. }
$$

Esto completa la demostración.

Nuestro objetivo es entonces probar la siguiente proposición:

Para cada $n \geq 2$ existe un impar $I<2 n$ primo relativo con $2 n$ tal que

$$
p=2 n+I, q=2 n-I
$$

son números primos.

Pero antes de esto es muy importante resaltar que el Postulado de Bertrand y el TEOREMA 1.5 son equivalentes es decir,

TEOREMA 1.6 Bertrand-Elías Para todo $n \geq 3$ existe un primo $p$ tal que $n<p<2 n \Leftrightarrow$ para todo $n \geq 3$ existe un impar $I\left(I_{1}\right)$ de tal menera que $2 n+I\left(2 n-I_{1}\right)$ es un número primo.

DEMOSTRACION $\Longleftarrow)$ Ya lo probamos. En el otro sentido. Sea $p$ un primo tal que $n<p<2 n$. Vemos que como $p<2 n$ existe un impar $I$ tal que $p+I=2 n$ es decir, $p=2 n-I$, el $I$ cambia según el $p$. Ahora, observemos que $n<p<2 n \Leftrightarrow$ $2 n<2 p<4 n$. Por hipótesis existe un primo $q$ tal que $2 n<$ $q<4 n$, luego existe $I_{1}$ impar talque $2 n=q-I_{1}$ Esto completa la prueba.

De otro lado si suponemos que

$$
p=2 n+I, q=2 n-I, \quad n \geq 2
$$

podemos observar lo siguiente:

- Sumando las dos ecuaciones encontramos

$$
p+q=4 n \text {. }
$$

- Restándolas: $p-q=2 I$.

- De otro lado si conocemos $q$ tenemos que $2 n=I+q$, sumando $I$ tenemos

$$
p=2 n+I=2 I+q .
$$


- Observemos que existen impares

$$
I_{1}<2 n, I_{2}<2 n,
$$

primos relativos con $2 n$ tales que $p=2 n+I_{1}$ y $q=2 n-I_{2}$ son números primos. Sumando tenemos

$$
p+q=4 n-\left(I_{2}-I_{1}\right)
$$

Si queremos que $p+q=2 n$ entonces, $I_{2}-I_{1}=2 n$. Como $p=2 n+I_{1}$ se tiene que $p=I_{2}$. Por lo que $q=2 n-p$. Es decir, $p+q=2 n$.

- Recordemos ahora que el postulado de Bertrand afirma que para cada $n \geq 3$ existe un primo $p$ tal que $n<p<2 n$. Al multiplicar por dos se encuentra $2 n<2 p<4 n$.

\section{Resultado principal}

Se trata de buscar un impar $1 \leq I \leq 2 n-3$ de tal manera que

$$
\text { (a) } \quad p=2 n+I, \quad q=2 n-I
$$

sean números primos. Para tal efecto notemos que

$$
\begin{aligned}
1 \leq I \leq & 2 n-3 \Leftrightarrow 2 n+1 \leq 2 n+I \leq 4 n-3 \\
& \Leftrightarrow 2 n+1 \leq p \leq 4 n-3
\end{aligned}
$$

De igual manera

$$
\begin{gathered}
1 \leq I \leq 2 n-3 \Leftrightarrow 3-2 n \leq-I \leq-1 \\
\Leftrightarrow 3 \leq 2 n-I \leq 2 n-1 \\
\Leftrightarrow 3 \leq q \leq 2 n-1
\end{gathered}
$$

Sumando las dos últimas desigualdades de $p, q$ encontramos

$$
2 n+4 \leq p+q \leq 6 n-4
$$

Si queremos que se cumpla (a) debe cumplirse que

$$
p+q=\frac{2 n+4+6 n-4}{2}=4 n
$$

Es decir, $p+q$ es el punto medio del intervalo

$$
[2 n+4,6 n-4]
$$

De otro lado, de (a) se deduce que $p=2 I+q$.

Dado el primo impar $q$ con $3 \leq q \leq 2 n-1$ (Nótese que $q$ es de la forma $2 n-I$ ) veamos que existe un primo $p$ tal que $p=2 I+q$ con $I$ impar. En efecto, como $2, q$ son primos relativos, la sucesión $p=2 m+q$ contiene infinitos números primos, elijamos un impar $I$ tal que $p=2 I+q$ sea primo y además $q$ primo relativo con $4 n$. Notemos que

$$
\begin{gathered}
p=2 I+q \Leftrightarrow 2 l=p-I=I+q \\
\Leftrightarrow p=2 l+I, q=2 l-I
\end{gathered}
$$

(suma y resta de impares es par). Es decir, para el par $2 l$ existe un impar $I$ que nos da el resultado deseado. Ahora, sumando las ecuaciones obtenidas encontramos que

$$
p+q=4 l=4 n \Rightarrow l=n
$$

Es decir, todo par de la forma $2 P, P=2 n$ se puede escribir como suma de dos números primos Aunque no se necesita, un razonamiento similar se puede hacer para el caso impar obteniendo una expresión de la forma $2 I=p+q$ con $p$ y $q$ números primos. Así tenemos los siguientes resultados:

COROLARIO 1.3. Elías- Primos Para cada $n \geq 2$ existe un impar $I<2 n$ primo relativo con $2 n$ tal que

$$
p=2 n+I, \quad q=2 n-I
$$

son números primos.

COROLARIO 1.4 Goldbach-Elías Todo par $P \geq 4$ se puede escribir como la suma de dos números primos.

Es decir, se ha demostrado la conjetura de Goldbach de una manera muy sencilla.

\section{CONCLUSIONES}

1. Se demuestra la Conjetura de Goldbach que ahora pasa a ser teorema.

2. Se generaliza el teorema de Dirichlet.

3. Se prueba el postulado de Bertrand de una manera sencilla.

4. El teorema de Dirichlet implica la conjetura de Goldbach.

\section{RECOMENDACIONES}

Motivar a estudiantes y docentes investigadores a utilizar herramientas sencillas en la demostración de ciertos resultados ya probados o sin probar con herramientas lo más sencillas posibles. 


\section{REFERENCIAS}

[1]. González Pineda C. E., Una nota sobre la conjetura de Goldbach. Scientia Et Technica, vol. XI, núm. 27, abril, 2005, pp. 213-214, Universidad Tecnológica de Pereira-Colombia.

[2]. González Pineda C. E., García S. M., Algunos tópicos en teoría de números: números Mersenne, teorema Dirichlet Números Fermat. Scientia Et Technica, vol. XVI, núm. 48, agosto, 2011, pp. 185-190,Universidad Tecnológica de Pereira-Colombia

[3]. Suárez M. F., Elementos de Álgebra. Centro Editorial. Universidad del Valle. 1994.

[4]. Ruiz, R. De los enteros a los dominios, Centro Editorial. Universidad del Valle 1994.

[5]. Niven, I. Zuckerman, H. S., Introducción a la Teoría de Números. Editorial Limusa-Wiley, México, 1969.

[6]. Jones W., Teoría de los Números Burton. Centro Regional para la ayuda técnica, Agencia para el desarrollo Internacional, México, 1969.

[7]. Shanks, D. (2001). Solved and unsolved problems in number theory (Vol. 297). American Mathematical Soc. 